\title{
REDUCIBILITY OF JOINS INVOLVING SOME LOCALLY TRIVIAL PSEUDOVARIETIES
}

\author{
J. C. Costa $^{1}$ \\ Centro de Matemática da Universidade do Minho \\ Campus de Gualtar, 4700-320 Braga, Portugal \\ Email: jcosta@math.uminho.pt
}

February 18, 2003

\begin{abstract}
In this paper, we show that $\sigma$-reducibility is preserved under joins with $\mathbf{K}$, where $\mathbf{K}$ is the pseudovariety of semigroups in which idempotents are left zeros. Reducibility of joins with $\mathbf{D}$, the pseudovariety of semigroups in which idempotents are right zeros, is also considered. In this case, we were able to prove that $\sigma$-reducibility is preserved for joins with pseudovarieties verifying a certain property of cancellation. As an example involving the semidirect product, we prove that $\mathbf{S l} * \mathbf{K}$ is $\kappa$-tame, where Sl stands for the pseudovariety of semilattices.
\end{abstract}

\section{INTRODUCTION}

As one recalls, the join $\mathbf{V} \vee \mathbf{W}$ of two pseudovarieties $\mathbf{V}$ and $\mathbf{W}$ is the least pseudovariety containing both $\mathbf{V}$ and $\mathbf{W}$. Decidability is not preserved by the join operator [1] neither by some of the most common operators on pseudovarieties [14]. In particular, Rhodes [14] has exhibited a decidable pseudovariety $\mathbf{V}$, defined by a finite set of identities, such that $\mathbf{S l} * \mathbf{V}$ is not decidable. Tameness is a property of pseudovarieties, introduced by Almeida and Steinberg [6], stronger than decidability, which may be important to prove decidability of pseudovarieties constructed using those operators. If tameness is preserved by the most common operators is still an open problem. As an example, not so difficult, we prove the tameness of the semidirect product $\mathbf{S l} * \mathbf{K}$, with respect to the canonical signature $\kappa$ consisting of multiplication together with the implicit operation $x \mapsto x^{\omega-1}$. A much more difficult case is the pseudovariety $\mathbf{S l} * \mathbf{D}$, whose $\kappa$-tameness was announced by Teixeira in collaboration with the author [11]. Notice that the pseudovarieties $\mathbf{K}, \mathbf{D}[9]$ and $\mathbf{S l}$ are tame.

Let $\sigma$ be an implicit signature. Proving the $\sigma$-tameness of a pseudovariety $\mathbf{V}$ involves proving two properties: that the $\sigma$-word problem for $\mathbf{V}$ is decidable, and that $\mathbf{V}$ is $\sigma$-reducible. This paper is concerned with the $\sigma$-reducibility of pseudovariety joins of the form $\mathbf{V} \vee \mathbf{W}$, where $\mathbf{V}$ is one of the pseudovarieties $\mathbf{K}, \mathbf{D}, \mathbf{N}$ or $\mathbf{L I}$, where $\mathbf{N}$ and LI are respectively the pseudovarieties of all nilpotent and locally trivial semigroups. These joins were studied by the author in [10], where they were computed for several pseudovarieties $\mathbf{V}$. Here, we prove that $\sigma$-reducibility is preserved for joins under $\mathbf{K}$ or $\mathbf{N}$; that is, if $\mathbf{W}$ is a $\sigma$-reducible pseudovariety, then $\mathbf{K} \vee \mathbf{W}$ and $\mathbf{N} \vee \mathbf{W}$ are also $\sigma$ reducible. We deduce in particular that $\mathbf{K} \vee \mathbf{W}$ and $\mathbf{N} \vee \mathbf{W}$ are $\kappa$-tame for every $\kappa$-tame pseudovariety $\mathbf{W}$. The study of the $\sigma$-reducibility of joins with $\mathbf{D}$ is much more difficult

\footnotetext{
${ }^{1}$ This work was supported, in part, by FCT through the Centro de Matemática da Universidade do Minho, and by the FCT and POCTI approved project POCTI/32817/MAT/2000 which is comparticipated by the European Community Fund FEDER.
} 
and we were not able to obtain a general answer. We prove that $\mathbf{D} \vee \mathbf{V}$ is $\sigma$-reducible for $\sigma$-reducible pseudovarieties $\mathbf{V}$ verifying a certain property of cancellation. We then derive some results for $\mathbf{L I}$.

The fundamental idea in our proofs is very simple. It consists in applying the $\sigma$ reducibility of $\mathbf{W}$ in such a way that the properties which determine the equality over $\mathbf{V}$ are preserved. The same techniques are explored in a forthcoming paper of the author in collaboration with Zeitoun [12] where we establish analogous results for the pseudovarieties $\mathbf{J}$ and $\mathbf{R}$ of $\mathcal{J}$-trivial and $\mathcal{R}$-trivial semigroups respectively.

This work is organized as follows. In a section of preliminaries, we recall the usual terminology and notation about words, pseudovarieties, graphs and $\sigma$-reducibility and review some basic results. Section 3 is devoted to the reducibility problem for joins with $\mathbf{K}$. We next prove that $\mathbf{S l} * \mathbf{K}$ is $\kappa$-tame. Finally, Section 5 is dedicated to the reducibility problem for joins with $\mathbf{D}$.

\section{PRELIMINARIES}

This section presents a brief description of the basic definitions, notation and results that are relevant to our study. The reader is referred to [2] for general background and missing definitions on pseudovarieties and profinite semigroups, and to [4] for recent developments on the subject, including the notion of tameness.

\subsection{Words}

An alphabet is a finite non-empty set $A$, whose elements are called letters. The free semigroup (resp. free monoid, free group) generated by $A$ is denoted by $A^{+}$(resp. $A^{*}$, $F G(A))$. The content $c(u)$ of a word $u \in A^{+}$is the set of all letters occurring in $u$ and the length of $u$ is denoted by $|u|$. The empty word is denoted by 1 .

A right-infinite (resp. left-infinite) word on $A$ is a sequence $w=\left(a_{n}\right)_{n}$ of letters of $A$ indexed by $\mathbb{N}$ (resp. $-\mathbb{N}$ ), also written

$$
w=a_{1} a_{2} a_{3} \cdots \quad\left(\text { resp. } w=\cdots a_{-3} a_{-2} a_{-1}\right) .
$$

The sets of right-infinite and left-infinite words on $A$ will be denoted, respectively, by $A^{\mathbb{N}}$ and $A^{-\mathbb{N}}$. We denote also $A^{\infty}=A^{+} \cup A^{\mathbb{N}}$ and $A^{-\infty}=A^{+} \cup A^{-\mathbb{N}}$. The product of two elements $w$ and $z$ of $A^{\infty}$ is defined as follows: if $w, z \in A^{*}$, then $w z$ is defined as usual; right-infinite words are left zeros; finally, if $w=a_{1} a_{2} \cdots a_{n}\left(a_{i} \in A\right)$ is a finite word and $z=b_{1} b_{2} \cdots\left(b_{j} \in A\right)$ is a right-infinite word, then $w z$ is the right-infinite word $w z=a_{1} a_{2} \cdots a_{n} b_{1} b_{2} \cdots$. The product of two elements of $A^{-\infty}$ is defined symmetrically. Now, we observe that $A^{\mathbb{N}}, A^{-\mathbb{N}}, A^{\infty}$ and $A^{-\infty}$ are semigroups.

A word $u \in A^{*}$ is a prefix of a word $w \in A^{\infty}$ if there exists $z \in A^{\infty}$ such that $w=u z$. Symmetrically, $u \in A^{*}$ is a suffix of $w \in A^{-\infty}$ if there exists $z \in A^{-\infty}$ such that $w=z u$.

A right-infinite word of the form $v u^{+\infty}=v u u u \cdots$, with $u \in A^{+}$and $v \in A^{*}$, is said to be ultimately periodic. Dually, we will use the notation $u^{-\infty} v$ to represent the ultimately periodic left-infinite word $u^{-\infty} v=\cdots u u u v$.

Two finite words $w$ and $z$ are said to be conjugate if there exist words $u, v \in A^{*}$ such that $w=u v$ and $z=v u$.

\subsection{Pseudovarieties and implicit operations}

For a semigroup $S$, we denote by $S^{1}$ the smallest monoid containing $S$. Given an element $s$ of a finite semigroup (resp. compact topological semigroup), the subsemigroup (resp. closed subsemigroup) generated by $s$ has a unique idempotent, denoted by $s^{\omega}$. Moreover, $s^{\omega-1}$ denotes the inverse of $s^{\omega+1}=s^{\omega} s$ in the maximal subgroup (resp. closed subgroup) containing $s$. As usual, we denote by $E(S)$ the set of idempotents of a semigroup $S$.

The next classical result follows immediately from [2, Proposition 3.7.1]. 
Lemma 2.1. Let $S$ be a finite semigroup and $k=|S|$. For any $s_{1}, \ldots, s_{k} \in S$, there exist integers $1 \leq i \leq j \leq k$, such that, $s_{1} \cdots s_{k}=s_{1} \cdots s_{i-1}\left(s_{i} \cdots s_{j}\right)^{\omega} s_{j+1} \cdots s_{k}$.

A semigroup pseudovariety is a class of finite semigroups closed under taking subsemigroups, homomorphic images and finite direct products. A profinite semigroup is a projective limit of finite semigroups. For a pseudovariety $\mathbf{V}$, a pro- $\mathbf{V}$ semigroup is a projective limit of semigroups of $\mathbf{V}$. The $A$-generated elements of $\mathbf{V}$ form a directed system and the respective projective limit will be denoted by $\bar{\Omega}_{A} \mathbf{V}$. The semigroup $\bar{\Omega}_{A} \mathbf{V}$ is a pro- $\mathbf{V}$ semigroup which is relatively free with respect to $\mathbf{V}$ in the sense that, for each pro- $\mathbf{V}$ semigroup $S$ and each mapping $\varphi: A \longrightarrow S$, there is a unique continuous morphism $\bar{\varphi}: \bar{\Omega}_{A} \mathbf{V} \longrightarrow S$ extending $\varphi$. This leads to a natural interpretation of elements of $\bar{\Omega}_{A} \mathbf{V}$ as (A-ary) implicit operations: to each $\pi \in \bar{\Omega}_{A} \mathbf{V}$ is associated an operation $\pi_{S}: S^{A} \longrightarrow S$ which maps $\varphi \in S^{A}$ to $\bar{\varphi}(\pi)$. The subsemigroup of $\bar{\Omega}_{A} \mathbf{V}$ generated by $A$, denoted by $\Omega_{A} \mathbf{V}$, is a dense subsemigroup of $\bar{\Omega}_{A} \mathbf{V}$ whose elements are said to be explicit. For an implicit operation $\pi$ of finite arity $A$, if we fix an ordering $a_{1}, \ldots, a_{r}$ for the elements of $A$, we also write $\pi=\pi\left(a_{1}, \ldots, a_{r}\right)$. As important examples of implicit operations we mention the (binary) operation of multiplication and the two (unary) operations $x \mapsto x^{\omega}$ and $x \mapsto x^{\omega-1}$. Notice that the composition of implicit operations is also an implicit operation.

The pseudovariety of all finite semigroups is denoted by $\mathbf{S}$ and, for each pseudovariety $\mathbf{V}, q_{\mathbf{V}}: \bar{\Omega}_{A} \mathbf{S} \longrightarrow \bar{\Omega}_{A} \mathbf{V}$ denotes the continuous morphism mapping the generators of $\bar{\Omega}_{A} \mathbf{S}$ to the generators of $\bar{\Omega}_{A} \mathbf{V}$. In particular, $q_{\mathbf{S l}}$ is usually denoted by $c$. Notice that $\bar{\Omega}_{A} \mathbf{S l}$ is $\mathcal{P}(A)$, the power set of $A$, under union. The morphism $c$ is called the content morphism and, as one recalls, $c$ extends to implicit operations the notion of content for words of $A^{+}$.

A pseudoidentity is a formal equality $\pi=\rho$ where $\pi, \rho \in \bar{\Omega}_{A} \mathbf{S}$. A finite semigroup $S$ satisfies a pseudoidentity $\pi=\rho$ if $\pi_{S}=\rho_{S}$. A pseudovariety $\mathbf{V}$ satisfies a pseudoidentity $\pi=\rho$ if every semigroup in $\mathbf{V}$ satisfies $\pi=\rho$, which means that $q_{\mathbf{V}}(\pi)=q_{\mathbf{V}}(\rho)$. By Reiterman's theorem [13], each pseudovariety is defined by a set $\Sigma$ of pseudoidentities. The pseudovariety defined by $\Sigma$ is denoted by $\llbracket \Sigma \rrbracket$.

We recall a result (see [2, Corollary 5.6.2]) which presents an useful decomposition of the non-explicit operations and constitutes a sort of analogue of Lemma 2.1.

Lemma 2.2. Let $\pi$ be a non-explicit element of $\bar{\Omega}_{A} \mathbf{S}$. Then, there exist $\pi_{1}, \rho, \pi_{2} \in \bar{\Omega}_{A} \mathbf{S}$ such that $\pi=\pi_{1} \rho^{\omega} \pi_{2}$.

\subsection{Graphs}

A (directed) graph $\Gamma$ is a set $\mathcal{V}(\Gamma) \stackrel{\circ}{\mathcal{E}}(\Gamma)$ with two sorts of elements, called vertices and edges respectively, together with two operations $\alpha, \omega: \mathcal{E}(\Gamma) \rightarrow \mathcal{V}(\Gamma)$ which define the orientation of the edges. For any edge $\mathrm{e} \in \mathcal{E}(\Gamma), \alpha(\mathrm{e})$ and $\omega(\mathrm{e})$ are, respectively, the beginning and the end of e.

A (directed) path in $\Gamma$ is a finite sequence of edges $\mathrm{e}_{1}, \mathrm{e}_{2}, \ldots, \mathrm{e}_{n}$ such that $\omega\left(\mathrm{e}_{i}\right)=$ $\alpha\left(\mathrm{e}_{i+1}\right)$ for $i=1, \ldots, n-1$. The path is a circuit if $\alpha\left(\mathrm{e}_{1}\right)=\omega\left(\mathrm{e}_{n}\right)$. Given a sequence of edges $e_{1}, \ldots, e_{n}$, if it is possible to invert the orientation of some edges in such a way to obtain a directed path, we say that $\mathrm{e}_{1}, \ldots, \mathrm{e}_{n}$ is an undirected path. By similarity, an undirected path is an undirected circuit if it is possible to invert the orientation of some edges in such a way to obtain a circuit.

A labelling of a graph $\Gamma$ by a semigroup $S$ is a mapping $\delta: \Gamma \longrightarrow S^{1}$ such that $\delta(\mathcal{E}(\Gamma)) \subseteq$ $S$. The label of a path $\mathrm{e}_{1}, \ldots, \mathrm{e}_{n}$ is by definition $\delta\left(\mathrm{e}_{1}\right) \cdots \delta\left(\mathrm{e}_{n}\right)$. A labelling $\delta$ is consistent if $\delta(\alpha(\mathrm{e})) \cdot \delta(\mathrm{e})=\delta(\omega(\mathrm{e}))$, for all $\mathrm{e} \in \mathcal{E}(\Gamma)$. If $\gamma: \mathcal{E}(\Gamma) \longrightarrow A^{+}$is a function, the label of an undirected path $\mathrm{e}_{1}, \ldots, \mathrm{e}_{n}$ is the reduced form of the word $\gamma\left(\mathrm{e}_{1}\right)^{\epsilon_{1}} \cdots \gamma\left(\mathrm{e}_{n}\right)^{\epsilon_{n}}$ in the free group generated by $A$, where $\epsilon_{i}=1$ if the edge $\mathrm{e}_{i}$ is read in the direct way, in the undirected path, and $\epsilon_{i}=-1$ otherwise. We say that the function $\gamma$ commutes if the label of any undirected circuit is 1 . 


\section{4. $\sigma$-reducibility and $\sigma$-tameness}

An implicit signature $\sigma$ is a set of implicit operations on $\mathbf{S}$ containing the basic semigroup multiplication. Every profinite semigroup has a natural structure of a $\sigma$ semigroup, via the interpretation of implicit operations as operations on profinite semigroups. For a pseudovariety $\mathbf{V}$, we denote by $\Omega_{A}^{\sigma} \mathbf{V}$ the free $\sigma$-semigroup generated by $A$ in the variety of $\sigma$-semigroups generated by $\mathbf{V}$, which as one can observe is the $\sigma$ subsemigroup of $\bar{\Omega}_{A} \mathbf{V}$ generated by $A$. The elements of $\Omega_{A}^{\sigma} \mathbf{S}$ are called $\sigma$-terms. We denote by $p_{\mathbf{V}}: \Omega_{A}^{\sigma} \mathbf{S} \longrightarrow \Omega_{A}^{\sigma} \mathbf{V}$ the morphism of $\sigma$-semigroups determined by the choice of generators. That is, $p_{\mathbf{V}}$ is the restriction of $q_{\mathbf{V}}$ to $\Omega_{A}^{\sigma} \mathbf{S}$. The $\sigma$-word problem for $\mathbf{V}$ is the problem of deciding equality of $\sigma$-terms in $\mathbf{V}$.

A labelling $\gamma: \Gamma \longrightarrow S^{1}$ of a finite graph $\Gamma$ by a finite $A$-generated semigroup $S$ is $\mathbf{V}$-inevitable if there exists a labelling $\delta$ of $\Gamma$ by $\bar{\Omega}_{A} \mathbf{S}$ such that $\psi \circ \delta=\gamma$, where the morphism $\psi: \bar{\Omega}_{A} \mathbf{S} \rightarrow S$ respects the choice of generators, and $q_{\mathbf{V}} \circ \delta$ is consistent. The pseudovariety $\mathbf{V}$ is said to be $\sigma$-reducible if for each $\mathbf{V}$-inevitable labelling $\gamma$, and $\delta$ and $\psi$ as above, there exists a labelling $\delta^{\prime}$ such that $\psi \circ \delta^{\prime}=\gamma$ and $p_{\mathbf{V}} \circ \delta^{\prime}$ is consistent.

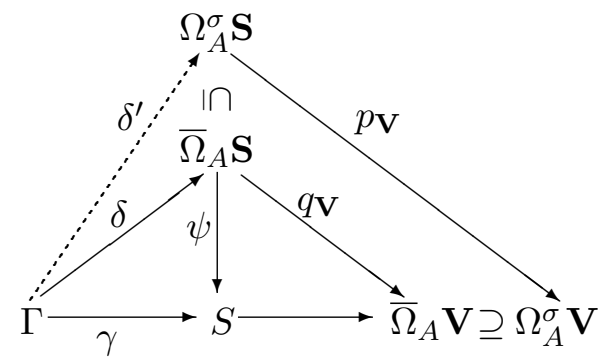

FiguRE 1. A commuting diagram expressing $\sigma$-reducibility

A recursively enumerable pseudovariety $\mathbf{V}$ is said to be $\sigma$-tame if it is $\sigma$-reducible and the $\sigma$-word problem for $\mathbf{V}$ is decidable. The next observation is crucial in what follows.

Lemma 2.3. Let $\mathbf{V}$ be a $\sigma$-reducible pseudovariety for a non-trivial (i.e., containing a non-explicit operation) implicit signature $\sigma$, let $\gamma: \Gamma \longrightarrow S^{1}$ be a labelling of a finite graph $\Gamma$ by a finite A-generated semigroup $S$ and let $\delta: \Gamma \rightarrow \bar{\Omega}_{A} \mathbf{S}$ be such that $\psi \circ \delta=\gamma$ and $q_{\mathbf{V}} \circ \delta$ is consistent, where the morphism $\psi: \bar{\Omega}_{A} \mathbf{S} \rightarrow S$ respects the choice of generators. Then, there is a labelling $\delta^{\prime}: \Gamma \rightarrow \Omega_{A}^{\sigma} \mathbf{S}$ such that,

i) $\psi \circ \delta^{\prime}=\gamma$ and $p_{\mathbf{V}} \circ \delta^{\prime}$ is consistent;

ii) if $\mathrm{e} \in \mathcal{E}(\Gamma)$ is an edge labelled under $\delta$ by a non-explicit operation, then $\delta^{\prime}(\mathrm{e})$ is also non-explicit;

iii) if $\mathrm{g} \in \Gamma$ is labelled under $\delta$ by an explicit operation, then $\delta^{\prime}(\mathrm{g})=\delta(\mathrm{g})$.

Proof. By Lemma 2.2, for each edge e such that $\delta(\mathrm{e})$ is non-explicit, there exist $\pi_{\mathrm{e}, 1}, \pi_{\mathrm{e}, 2}, \rho_{\mathrm{e}} \in \bar{\Omega}_{A} \mathbf{S}$ such that $\delta(\mathrm{e})=\pi_{\mathrm{e}, 1} \rho_{\mathrm{e}}^{\omega} \pi_{\mathrm{e}, 1}$. For each such e, add to graph $\Gamma$ a new vertex $w_{e}$ and three new edges $f_{e, 1}$ (from $\alpha(e)$ to $\left.w_{e}\right), f_{e, 2}\left(\right.$ from $w_{e}$ to $w_{e}$ ) and $f_{e, 3}$ (from $w_{\mathrm{e}}$ to $\omega(\mathrm{e})$ ). The graph obtained is denoted by $\Gamma_{1}$. Let $\delta_{1}$ be the extension of $\delta$ to $\Gamma_{1}$ such that $\delta_{1}\left(\mathrm{w}_{\mathrm{e}}\right)=\delta(\alpha(\mathrm{e})) \cdot \pi_{\mathrm{e}, 1} \rho_{\mathrm{e}}^{\omega}, \delta_{1}\left(\mathrm{f}_{\mathrm{e}, 1}\right)=\pi_{\mathrm{e}, 1} \rho_{\mathrm{e}}^{\omega}, \delta_{1}\left(\mathrm{f}_{\mathrm{e}, 2}\right)=\rho_{\mathrm{e}}^{\omega}$ and $\delta_{1}\left(\mathrm{f}_{\mathrm{e}, 3}\right)=\pi_{\mathrm{e}, 2}$. Since $q_{\mathbf{V}} \circ \delta$ is consistent, it is clear that $q_{\mathbf{V}} \circ \delta_{1}$ is also consistent.

Let now $m$ be an integer greater than the maximal length of the labels of $\Gamma$ under $\delta$ which are explicit, let $\mathbf{K}_{m}=\llbracket x_{1} x_{2} \cdots x_{m} y=x_{1} x_{2} \cdots x_{m} \rrbracket$ and let $S_{1}$ be the semigroup $S \times K_{m}$ where $K_{m}=\bar{\Omega}_{A} \mathbf{K}_{m}$. The semigroup $S_{1}$ is finite and $A$-generated, since $K_{m}$ may be seen as the set of all words on the alphabet $A$ of length at most $m$, where the product of two words is the longest prefix of length at most $m$ of their usual product. Let $\psi_{1}: \bar{\Omega}_{A} \mathbf{S} \rightarrow S_{1}$ be the morphism defined, for each $\pi \in \bar{\Omega}_{A} \mathbf{S}$, by $\psi_{1}(\pi)=\left(\psi(\pi), q_{\mathbf{K}_{m}}(\pi)\right)$, and let $\gamma_{1}: \Gamma_{1} \rightarrow S_{1}$ be the labelling of $\Gamma_{1}$ by $S_{1}$ such that $\gamma_{1}=\psi_{1} \circ \delta_{1}$. Hence, 
since $\psi_{1}$ respects the choice of generators and $q_{\mathbf{V}} \circ \delta_{1}$ is consistent, $\gamma_{1}$ is a $\mathbf{V}$-inevitable labelling of $\Gamma_{1}$ by $S_{1}$. Therefore, since $\mathbf{V}$ is $\sigma$-reducible there exists a labelling $\delta_{1}^{\prime}$ of $\Gamma_{1}$ by $\Omega_{A}^{\sigma} \mathbf{S}$ such that $\psi_{1} \circ \delta_{1}^{\prime}=\gamma_{1}$ and $p_{\mathbf{V}} \circ \delta_{1}^{\prime}$ is consistent. This implies in particular that $\psi_{1} \circ \delta_{1}^{\prime}=\psi_{1} \circ \delta_{1}$, whence $\psi \circ \delta_{1}^{\prime}=\psi \circ \delta_{1}$ and $q_{\mathbf{K}_{m}} \circ \delta_{1}^{\prime}=q_{\mathbf{K}_{m}} \circ \delta_{1}$.

For each edge e such that $\delta(\mathrm{e})$ is non-explicit and each $i \in\{1,2,3\}$, let $t_{\mathrm{e}, i}=\delta_{1}^{\prime}\left(\mathrm{f}_{\mathrm{e}, i}\right)$. By consistency of $p_{\mathbf{V}} \circ \delta_{1}^{\prime}, \mathbf{V}$ satisfies

$$
\delta_{1}^{\prime}(\omega(\mathrm{e}))=\delta_{1}^{\prime}(\alpha(\mathrm{e})) t_{\mathrm{e}, 1} t_{\mathrm{e}, 3}=\delta_{1}^{\prime}(\alpha(\mathrm{e})) t_{\mathrm{e}, 1} t_{\mathrm{e}, 2}^{n} t_{\mathrm{e}, 3}
$$

for every positive integer $n$. On the other hand, since $\psi$ is a morphism and verifies $\psi \circ \delta_{1}^{\prime}=\psi \circ \delta_{1}$,

$$
\psi\left(t_{\mathrm{e}, 1} t_{\mathrm{e}, 2}^{n} t_{\mathrm{e}, 3}\right)=\psi\left(t_{\mathrm{e}, 1}\right) \psi\left(t_{\mathrm{e}, 2}\right)^{n} \psi\left(t_{\mathrm{e}, 3}\right)=\psi\left(\pi_{\mathrm{e}, 1} \rho_{\mathrm{e}}^{\omega}\right) \psi\left(\rho_{\mathrm{e}}^{\omega}\right)^{n} \psi\left(\pi_{\mathrm{e}, 2}\right)=\psi\left(\pi_{\mathrm{e}, 1} \rho_{\mathrm{e}}^{\omega} \pi_{\mathrm{e}, 2}\right) .
$$

Therefore, since $\psi \circ \delta=\gamma$,

$$
\psi\left(t_{\mathrm{e}, 1} t_{\mathrm{e}, 2}^{n} t_{\mathrm{e}, 3}\right)=\psi(\delta(\mathrm{e}))=\gamma(\mathrm{e}) .
$$

Let now $\varrho\left(a_{1}, \ldots, a_{r}\right)$ be a non-explicit element of the implicit signature $\sigma$ and let $\left(w_{i}\left(a_{1}, \ldots, a_{r}\right)\right)_{i}$ be a sequence of explicit operations converging to $\varrho\left(a_{1}, \ldots, a_{r}\right)$. Then $\left(w_{i}\left(t_{\mathrm{e}, 2}, \ldots, t_{\mathrm{e}, 2}\right)\right)_{i}$ is a sequence which converges to the non-explicit $\sigma$-term $\varrho\left(t_{\mathrm{e}, 2}, \ldots, t_{\mathrm{e}, 2}\right)$. Since for each $i$ there exists an integer $n_{i}$ such that $w_{i}\left(t_{\mathrm{e}, 2}, \ldots, t_{\mathrm{e}, 2}\right)=t_{\mathrm{e}, 2}^{n_{i}}$, we deduce from (2) that the non-explicit $\sigma$-term $t_{\mathrm{e}}=t_{\mathrm{e}, 1} \varrho\left(t_{\mathrm{e}, 2}, \ldots, t_{\mathrm{e}, 2}\right) t_{\mathrm{e}, 3}$ is such that $\psi\left(t_{\mathrm{e}}\right)=\gamma(\mathrm{e})$. Moreover, by $(1), p_{\mathbf{V}}\left(\delta_{1}^{\prime}(\omega(\mathrm{e}))\right)=p_{\mathbf{V}}\left(\delta_{1}^{\prime}(\alpha(\mathrm{e}))\right) p_{\mathbf{V}}\left(t_{\mathrm{e}}\right)$. Let $\delta^{\prime}$ coincide with $\delta_{1}^{\prime}$ on $\Gamma \backslash\{\mathrm{e} \mid \delta(\mathrm{e})$ is non-explicit $\}$ and let $\delta^{\prime}(\mathrm{e})=t_{\mathrm{e}}$ for each edge e such that $\delta(\mathrm{e})$ is non-explicit. Therefore $\delta^{\prime}$ is a labelling of $\Gamma$ by $\Omega_{A}^{\sigma} \mathbf{S}$ verifying i) and ii).

Now, let $\mathrm{g} \in \Gamma$ be such that $\delta(\mathrm{g})$ is explicit. Then

$$
\begin{aligned}
q_{\mathbf{K}_{m}} \circ \delta^{\prime}(\mathrm{g}) & =q_{\mathbf{K}_{m}} \circ \delta_{1}^{\prime}(\mathrm{g}) \quad \text { by definition of } \delta^{\prime} \\
& =q_{\mathbf{K}_{m}} \circ \delta_{1}(\mathrm{~g}) \quad \text { since } q_{\mathbf{K}_{m}} \circ \delta_{1}^{\prime}=q_{\mathbf{K}_{m}} \circ \delta_{1} \\
& =q_{\mathbf{K}_{m}} \circ \delta(\mathrm{g}) \quad \text { since } \delta_{1} \text { and } \delta \text { coincide on } \Gamma
\end{aligned}
$$

Since $\delta(\mathrm{g})$ is a word of length at most $m-1$, we deduce that $q_{\mathbf{K}_{m}} \circ \delta^{\prime}(\mathrm{g})=\delta(\mathrm{g})$, whence $\delta^{\prime}(\mathrm{g})=\delta(\mathrm{g})$. This proves iii) and concludes the proof of the lemma.

\subsection{Basic Lemmas}

This section gathers some basic statements about the free pro- $\mathbf{V}$ semigroups, with $\mathbf{V} \in\{\mathbf{N}, \mathbf{K}, \mathbf{D}, \mathbf{L I}, \mathbf{S l} * \mathbf{K}\}$, which will be used in this work. Recall first that

$$
\begin{aligned}
& \mathbf{N}=\llbracket x^{\omega} y=x^{\omega}=y x^{\omega} \rrbracket, \quad \mathbf{K}=\llbracket x^{\omega} y=x^{\omega} \rrbracket, \quad \mathbf{D}=\llbracket y x^{\omega}=x^{\omega} \rrbracket \\
& \mathbf{L I}=\llbracket x^{\omega} y z^{\omega}=x^{\omega} z^{\omega} \rrbracket, \quad \mathbf{S} \mathbf{l} * \mathbf{K}=\llbracket x^{\omega} y^{2}=x^{\omega} y, x^{\omega} y z=x^{\omega} z y \rrbracket .
\end{aligned}
$$

Notice also that $\mathbf{N}=\mathbf{K} \cap \mathbf{D}, \mathbf{L I}=\mathbf{K} \vee \mathbf{D}$ and $\mathbf{K} \subseteq \mathbf{S l} * \mathbf{K}$. The following properties are well known (see [2], for instance).

Lemma 2.4. Let $\mathbf{V}$ be one of the pseudovarieties $\mathbf{N}, \mathbf{K}$ or $\mathbf{D}$. Then, $\Omega_{A} \mathbf{V}$ is isomorphic to $A^{+}$and $E\left(\bar{\Omega}_{A} \mathbf{V}\right)$ is an ideal consisting of the non-explicit operations of $\bar{\Omega}_{A} \mathbf{V}$. Moreover,

i) The unique idempotent of $\bar{\Omega}_{A} \mathbf{N}$ is a zero;

ii) $E\left(\bar{\Omega}_{A} \mathbf{K}\right)$ is isomorphic to $A^{\mathbb{N}}$, and $\bar{\Omega}_{A} \mathbf{K}$ is isomorphic to $A^{\infty}$;

iii) $E\left(\bar{\Omega}_{A} \mathbf{D}\right)$ is isomorphic to $A^{-\mathbb{N}}$, and $\bar{\Omega}_{A} \mathbf{D}$ is isomorphic to $A^{-\infty}$.

In view of this lemma, we shall identify explicit operations (on $\mathbf{N}, \mathbf{K}$ or $\mathbf{D}$ ) with finite words. Moreover, the idempotents of $\bar{\Omega}_{A} \mathbf{K}$ and $\bar{\Omega}_{A} \mathbf{D}$ may be identified, respectively, with right-infinite words and left-infinite words. Notice that in $\bar{\Omega}_{A} \mathbf{K}$, the right-infinite word $y x^{+\infty}$, where $y \in A^{*}$ and $x \in A^{+}$, corresponds to the implicit operation $y x^{\omega}$. A dual remark holds for $\mathbf{D}$.

A proof of the following useful result can be found in [9]. 
Lemma 2.5. Let $\pi, \rho \in \bar{\Omega}_{A} \mathbf{S}$ be non-explicit operations such that $q_{\mathbf{D}}(\pi)=q_{\mathbf{D}}(\rho)$. Then there exist factorizations $\pi=\pi_{1} \pi_{2}$ and $\rho=\rho_{1} \pi_{2}$ where $\pi_{2}$ is non-explicit. Moreover, if $q_{\mathbf{D}}(\pi)=u^{-\infty} v$ with $u \in A^{+}$and $v \in A^{*}$, then we can choose $\pi_{2}=u^{\omega} v$.

For an alphabet $A$ and an infinite word $v \in A^{\mathbb{N}}$, we let $c(v)$ be the set of letters occurring in $v$ and let $c_{\infty}(v)$ be the set of letters occurring infinitely often in $v$. Almeida and Weil [7] gave the description of the free pro- $(\mathbf{S l} * \mathbf{K})$ semigroups and solved the word problem for them. These results are presented in the next theorem.

Theorem 2.6. Let $A$ be an alphabet. Then

$$
\bar{\Omega}_{A}(\mathbf{S l} * \mathbf{K})=A^{+} \cup\left\{(v, B) \in A^{\mathbb{N}} \times \mathcal{P}(A) \mid c_{\infty}(v) \subseteq B\right\},
$$

and the product is given, for all $u, u^{\prime} \in A^{+}$and $(v, B),\left(v^{\prime}, B^{\prime}\right) \in A^{\mathbb{N}} \times \mathcal{P}(A)$ such that $c_{\infty}(v) \subseteq B$ and $c_{\infty}\left(v^{\prime}\right) \subseteq B^{\prime}$, by:

$$
\begin{aligned}
u \cdot u^{\prime} & =u u^{\prime} \\
u \cdot(v, B) & =(u v, B) \\
(v, B) \cdot u & =(v, B \cup c(u)) \\
(v, B) \cdot\left(v^{\prime}, B^{\prime}\right) & =\left(v, B \cup c\left(v^{\prime}\right) \cup B^{\prime}\right) .
\end{aligned}
$$

Each non-explicit operation $\pi \in \bar{\Omega}_{A}(\mathbf{S l} * \mathbf{K})$ admits a factorization of the form $\pi=$ $u_{0} \pi_{1} u_{1}$ where $u_{0}, u_{1} \in A^{*}, \pi_{1}$ is idempotent, the last letter of $u_{0}$ (if $u_{0}$ is non-empty) is not in $c\left(\pi_{1}\right)$, the word $u_{1}$ is linear (i.e. each letter appears at most once in $u_{1}$ ) and $c\left(u_{1}\right) \cap c\left(\pi_{1}\right)=\emptyset$. Furthermore, this factorization is unique up to a permutation of $u_{1}$. To be more precise, if $\pi=(v, B) \in A^{\mathbb{N}} \times \mathcal{P}(A)$, then $u_{0}$ is uniquely determined as the shortest prefix $u$ of $v$ such that $c\left(u^{-1} v\right)=c_{\infty}(v), \pi_{1}=\left(u_{0}^{-1} v, c_{\infty}(v)\right)$ and $c\left(u_{1}\right)=B \backslash c_{\infty}(v)$.

We will use also the following dual of Lemma 2.5.

Lemma 2.7. Let $\pi, \rho \in \bar{\Omega}_{A} \mathbf{S}$ be non-explicit operations such that $q_{\mathbf{K}}(\pi)=q_{\mathbf{K}}(\rho)=v$ and let $u \in A^{*}$ be any prefix of $v$. Then there exist factorizations $\pi=u \pi_{1} \pi_{2}$ and $\rho=u \pi_{1} \rho_{2}$ where $\pi_{1}$ is non-explicit. Moreover, $q_{\mathbf{S} \mathbf{1} * \mathbf{K}}(\pi)=q_{\mathbf{S} \mathbf{1} * \mathbf{K}}(\rho)$ if and only if $c_{\infty}(v) \cup c\left(\pi_{2}\right)=c_{\infty}(v) \cup c\left(\rho_{2}\right)$.

Proof. The first part of the lemma is an easy adaptation of the dual of Lemma 2.5. Notice that, in particular, $q_{\mathbf{K}}\left(u \pi_{1}\right)=v$. Therefore, the second part is an immediate consequence of Theorem 2.6 and of the fact that the content morphism is continuous. $\square$

\section{REDUCIBILITY OF JOINS WITH K}

In this section, we show that the property of being $\sigma$-reducible is preserved under joins with $\mathbf{K}$.

Theorem 3.1. If $\mathbf{V}$ is a $\sigma$-reducible pseudovariety for a non-trivial implicit signature $\sigma$, then $\mathbf{K} \vee \mathbf{V}$ is $\sigma$-reducible.

Proof. Let $\gamma$ be a $(\mathbf{K} \vee \mathbf{V})$-inevitable labelling of a finite graph $\Gamma$ by a finite $A$-generated semigroup $S$ and let $\psi$ be the unique morphism from $\bar{\Omega}_{A} \mathbf{S}$ to $S$ which respects the choice of generators. Then, there is a labelling $\delta$ of $\Gamma$ by $\bar{\Omega}_{A} \mathbf{S}$ such that $\psi \circ \delta=\gamma$ and $q_{\mathbf{K} \vee \mathbf{V}} \circ \delta$ is consistent. Notice that this implies that $q_{\mathbf{K}} \circ \delta$ and $q_{\mathbf{V}} \circ \delta$ are both consistent. We have to construct a labelling $\delta^{\prime}: \Gamma \rightarrow \Omega_{A}^{\sigma} \mathbf{S}$ such that $\psi \circ \delta^{\prime}=\gamma$ and $p_{\mathbf{K} \vee \mathbf{V}} \circ \delta^{\prime}$ is consistent. The beginning of the construction of $\delta^{\prime}$ will follow step by step the construction of Almeida and Zeitoun [9] for the corresponding labelling in the case of $\mathbf{K}$.

Let $\mathcal{V}_{\omega}$ be the set of vertices $v \in \mathcal{V}(\Gamma)$ such that $\delta(\mathrm{v})$ is a non-explicit operation and let $\mathcal{V}_{0}=\mathcal{V}(\Gamma) \backslash \mathcal{V}_{\omega}$. Let $\mathcal{E}_{\omega}$ be the set of edges e $\in \mathcal{E}(\Gamma)$ such that $\alpha(\mathrm{e}) \in \mathcal{V}_{\omega}$, and let $\mathcal{E}_{0}=\mathcal{E}(\Gamma) \backslash \mathcal{E}_{\omega}$. Let $\theta$ be the equivalence relation on $\mathcal{V}_{\omega}$ generated by the relation

$$
\left\{(\mathrm{v}, \mathrm{w}) \mid \mathrm{v}, \mathrm{w} \in \mathcal{V}_{\omega} \text { and there is an edge from } \mathrm{v} \text { to } \mathrm{w}\right\}
$$


and denote by $\theta(\mathrm{v})$ the $\theta$-class of v. Finally, let $\mathcal{E}_{\theta(\mathrm{v})}$ be the subset of $\mathcal{E}_{0}$ consisting of all edges e such that $\omega(\mathrm{e}) \in \theta(\mathrm{v})$.

Let $v \in \mathcal{V}_{\omega}$. Notice that, for every edge $\mathrm{e} \in \mathcal{E}_{\theta(\mathrm{v})}$, the label $\delta(\alpha(\mathrm{e}))$ is explicit and the label $\delta(\mathrm{e})$ is non-explicit. So we can consider the integer

$$
m_{\theta(\mathrm{v})}=\max \left\{|\delta(\alpha(\mathrm{e}))| \mid \mathrm{e} \in \mathcal{E}_{\theta(\mathrm{v})}\right\}
$$

and let, respectively, $p_{\theta(\mathrm{v})}$ be the word of length $m_{\theta(\mathrm{v})}$ and $w_{\theta(\mathrm{v})} \in \bar{\Omega}_{A} \mathbf{K}$ be the nonexplicit operation such that

$$
q_{\mathbf{K}}(\delta(\mathrm{v}))=p_{\theta(\mathrm{v})} w_{\theta(\mathrm{v})} .
$$

The choice of $m_{\theta(\mathrm{v})}$ and the fact that $q_{\mathbf{K}} \circ \delta$ is consistent, imply that $w_{\theta(\mathrm{v})}$ is also a suffix of $q_{\mathbf{K}}(\delta(\mathrm{e}))$, for every edge e $\in \mathcal{E}_{\theta(\mathrm{v})}$, so that

$$
q_{\mathbf{K}}(\delta(\mathrm{e}))=p_{\mathrm{e}} w_{\theta(\mathrm{v})}
$$

where $p_{\mathrm{e}}$ is the word such that

$$
\delta(\alpha(\mathrm{e})) \cdot p_{\mathrm{e}}=p_{\theta(\mathrm{v})}
$$

By Lemma 2.7, the factorizations (3) and (4) imply the existence of implicit operations $\pi_{\theta(\mathrm{v})}, \rho_{\mathrm{v}}$ and $\rho_{\mathrm{e}}$, with $\pi_{\theta(\mathrm{v})}$ non-explicit, such that

$$
\begin{aligned}
& \delta(\mathrm{v})=p_{\theta(\mathrm{v})} \cdot \pi_{\theta(\mathrm{v})} \cdot \rho_{\mathrm{v}}, \\
& \delta(\mathrm{e})=p_{\mathrm{e}} \cdot \pi_{\theta(\mathrm{v})} \cdot \rho_{\mathrm{e}} \text { for every edge } \mathrm{e} \in \mathcal{E}_{\theta(\mathrm{v})} .
\end{aligned}
$$

Notice that for a vertex $v \in \mathcal{V}_{\omega}$, if $\mathcal{E}_{\theta(\mathrm{v})}$ is the empty set, then we may choose for $p_{\theta(\mathrm{v})}$ any prefix of $q_{\mathbf{K}}(\delta(\mathrm{v}))$ and deduce a factorization of $\delta(\mathrm{v})$ analogous to (6).

We extend graph $\Gamma$ to a graph $\Gamma_{1}$ and let $\gamma_{1}$ and $\delta_{1}$ be respectively the extensions of $\gamma$ and $\delta$ to $\Gamma_{1}$ as follows. For each $\theta$-class $\Theta$,

- there are two vertices $w_{\Theta, 1}$ and $w_{\Theta, 2}$, and an edge $f_{\Theta}$ (from $w_{\Theta, 1}$ to $w_{\Theta, 2}$ ) labelled under $\delta_{1}$ by $p_{\Theta}, p_{\Theta} \pi_{\Theta}$ and $\pi_{\Theta}$, respectively;

- for each vertex $v \in \Theta$, there is an edge $f_{v}$ from $w_{\Theta, 2}$ to $v$ labelled under $\delta_{1}$ by $\rho_{v}$;

- for each edge $\mathrm{e} \in \mathcal{E}_{\Theta}$, there is an edge $\mathrm{f}_{\mathrm{e}}$ from $\mathrm{w}_{\Theta, 2}$ to $\omega(\mathrm{e})$ labelled under $\delta_{1}$ by $\rho_{\mathrm{e}}$;

- for each $\mathrm{g} \in \Gamma_{1} \backslash \Gamma, \gamma_{1}(\mathrm{~g})=\psi \circ \delta_{1}(\mathrm{~g})$.

Since $\psi \circ \delta=\gamma$ and $q_{\mathbf{K} \vee \mathbf{V}} \circ \delta$ is consistent, we deduce by construction that $\psi \circ \delta_{1}=\gamma_{1}$ and $q_{\mathbf{K} \vee \mathbf{V}} \circ \delta_{1}$ is consistent. In particular, $q_{\mathbf{V}} \circ \delta_{1}$ is consistent. Therefore, since $\mathbf{V}$ is $\sigma$-reducible there exists a labelling $\delta_{1}^{\prime}$ of $\Gamma_{1}$ by $\Omega_{A}^{\sigma} \mathbf{S}$ such that $\psi \circ \delta_{1}^{\prime}=\gamma_{1}$ and $p_{\mathbf{V}} \circ \delta_{1}^{\prime}$ is consistent. Moreover, by Lemma 2.3, we may assume that $\delta_{1}^{\prime}\left(\mathrm{f}_{\Theta}\right)$ is non-explicit for every $\theta$-class $\Theta$ and that $\delta_{1}^{\prime}(\mathrm{g})=\delta_{1}(\mathrm{~g})$ for every $\mathrm{g} \in \Gamma_{1}$ such that $\delta_{1}(\mathrm{~g})$ is explicit. Notice that, in particular, $\delta_{1}^{\prime}(\mathrm{g})=\delta(\mathrm{g})$ for every $\mathrm{g} \in \Gamma$ such that $\delta(\mathrm{g})$ is explicit.

We define $\delta^{\prime}$ to be the labelling of $\Gamma$ by $\Omega_{A}^{\sigma} \mathbf{S}$ as follows:

- $\delta^{\prime}$ coincides with $\delta$ on vertices and edges labelled by explicit operations;

- $\delta^{\prime}(\mathrm{e})=\delta_{1}^{\prime}(\mathrm{e})$ for any edge e $\in \mathcal{E}_{\omega}$;

- for each vertex $\mathrm{v} \in \mathcal{V}_{\omega}$ and each edge e $\in \mathcal{E}_{\theta(\mathrm{v})}, \delta^{\prime}(\mathrm{v})=p_{\theta(\mathrm{v})} \delta_{1}^{\prime}\left(\mathrm{f}_{\theta(\mathrm{v})}\right) \delta_{1}^{\prime}\left(\mathrm{f}_{\mathrm{v}}\right)$ and $\delta^{\prime}(\mathrm{e})=p_{\mathrm{e}} \delta_{1}^{\prime}\left(\mathrm{f}_{\theta(\mathrm{v})}\right) \delta_{1}^{\prime}\left(\mathrm{f}_{\mathrm{e}}\right)$.

By construction, $\psi \circ \delta^{\prime}=\gamma$. Indeed, let for instance $\vee \in \mathcal{V}_{\omega}$. We have

$$
\begin{aligned}
\psi \circ \delta^{\prime}(\mathrm{v}) & =\psi\left(p_{\theta(\mathrm{v})} \delta_{1}^{\prime}\left(\mathrm{f}_{\theta(\mathrm{v})}\right) \delta_{1}^{\prime}\left(\mathrm{f}_{\mathrm{v}}\right)\right) \\
& =\psi\left(p_{\theta(\mathrm{v})} \delta_{1}\left(\mathrm{f}_{\theta(\mathrm{v})}\right) \delta_{1}\left(\mathrm{f}_{\mathrm{v}}\right)\right) \quad \text { since } \psi \circ \delta_{1}^{\prime}=\psi \circ \delta_{1} \\
& =\psi\left(p_{\theta(\mathrm{v})} \pi_{\theta(\mathrm{v})} \rho_{\mathrm{v}}\right) \quad \text { by definition of } \delta_{1} \\
& =\psi \circ \delta(\mathrm{v}) \quad \text { by }(6) \\
& =\gamma(\mathrm{v}) .
\end{aligned}
$$

Moreover, since $p_{\mathbf{V}} \circ \delta_{1}^{\prime}$ is consistent one deduces that $p_{\mathbf{V}} \circ \delta^{\prime}$ is consistent. On the other hand $p_{\mathbf{K}} \circ \delta^{\prime}$ is also consistent. Indeed, let e be an edge of $\Gamma$ and let $v=\omega(\mathrm{e})$. 
We have to prove that $p_{\mathbf{K}}\left(\delta^{\prime}(\alpha(\mathrm{e}))\right) \cdot p_{\mathbf{K}}\left(\delta^{\prime}(\mathrm{e})\right)=p_{\mathbf{K}}\left(\delta^{\prime}(\mathrm{v})\right)$. The case in which $\delta(\mathrm{e})$ is explicit is immediate. If $\alpha(\mathrm{e}) \in \mathcal{V}_{\omega}$, then $\alpha(\mathrm{e})$ and $\vee$ belong both to the same $\theta$-class and since $\delta_{1}^{\prime}\left(\mathrm{f}_{\theta(\mathrm{v})}\right)$ is non-explicit, we deduce from the definition of $\delta^{\prime}(\alpha(\mathrm{e}))$ and $\delta^{\prime}(\mathrm{v})$ that the equality also holds in this case. Suppose now that e $\in \mathcal{E}_{\theta(\mathrm{v})}$, whence $\delta(\alpha(\mathrm{e}))$ is explicit and $\delta(\mathrm{e})$ is non-explicit. We have

$$
\begin{aligned}
& p_{\mathbf{K}}\left(\delta^{\prime}(\alpha(\mathrm{e}))\right) \cdot p_{\mathbf{K}}\left(\delta^{\prime}(\mathrm{e})\right)= \\
= & p_{\mathbf{K}}\left(\delta(\alpha(\mathrm{e})) p_{\mathrm{e}} \delta_{1}^{\prime}\left(\mathrm{f}_{\theta(\mathrm{v})}\right) \delta_{1}^{\prime}\left(\mathrm{f}_{\mathrm{e}}\right)\right) \\
= & p_{\mathbf{K}}\left(\delta(\alpha(\mathrm{e})) p_{\mathrm{e}} \delta_{1}^{\prime}\left(\mathrm{f}_{\theta(\mathrm{v})}\right) \delta_{1}^{\prime}\left(\mathrm{f}_{\mathrm{v}}\right)\right) \quad \text { since } \delta_{1}^{\prime}\left(\mathrm{f}_{\theta(\mathrm{v})}\right) \text { is non-explicit } \\
= & p_{\mathbf{K}}\left(\delta^{\prime}(\mathrm{v})\right) \text { by }(5) .
\end{aligned}
$$

Hence $p_{\mathbf{V}} \circ \delta^{\prime}$ and $p_{\mathbf{K}} \circ \delta^{\prime}$ are both consistent. Therefore $p_{\mathbf{K} \vee \mathbf{V}} \circ \delta_{1}^{\prime}$ is consistent. This concludes the proof of the theorem.

Now notice that if the $\sigma$-word problem is decidable for two pseudovarieties $\mathbf{V}$ and $\mathbf{W}$, then the $\sigma$-word problem for $\mathbf{V} \vee \mathbf{W}$ is decidable. Therefore, as a consequence of the last theorem, we deduce that $\sigma$-tameness is preserved under joins with $\mathbf{K}$.

Corollary 3.2. Let $\sigma$ be a non-trivial implicit signature. If $\mathbf{V}$ is a $\sigma$-tame pseudovariety and the $\sigma$-word problem for $\mathbf{K}$ is decidable, then $\mathbf{K} \vee \mathbf{V}$ is $\sigma$-tame.

The canonical signature $\kappa$ is the most commonly used signature: most of the pseudovarieties which are known to be tame are proved to be $\kappa$-tame. It is known, for instance, that $\mathbf{K}, \mathbf{D}[9], \mathbf{G}[6], \mathbf{A b}[5]$ and $\mathbf{J}[8]$ are $\kappa$-tame. Hence, for $\sigma=\kappa$, Corollary 3.2 can be stated as follows.

Corollary 3.3. If $\mathbf{V}$ is a $\kappa$-tame pseudovariety, then $\mathbf{K} \vee \mathbf{V}$ is $\kappa$-tame.

In particular, we have that $\mathbf{L I}=\mathbf{K} \vee \mathbf{D}, \mathbf{K} \vee \mathbf{G}, \mathbf{K} \vee \mathbf{A b}$ and $\mathbf{K} \vee \mathbf{J}$ are $\kappa$-tame.

The results of this section are easily shown to hold with $\mathbf{K}$ replaced by $\mathbf{N}$.

\section{TAMENESS OF $\mathbf{S l} * \mathbf{K}$}

In this section, we show that the semidirect product $\mathbf{S l} * \mathbf{K}$ is $\kappa$-tame. Since $\mathbf{S l} * \mathbf{K}$ is aperiodic, i.e., $\mathbf{S l} * \mathbf{K}$ verifies $x^{\omega}=x^{\omega+1}$, we use the implicit operation $x \mapsto x^{\omega}$ instead of $x \mapsto x^{\omega-1}$ since the two coincide over aperiodic semigroups and the former is expressible in terms of the latter.

\subsection{The $\kappa$-word problem for $\mathrm{Sl} * \mathbf{K}$}

We first observe that two $\kappa$-terms which do not involve the operation $x \mapsto x^{\omega}$ are equal over $\mathbf{S l} * \mathbf{K}$ if and only if they are the same term. Secondly, if two $\kappa$-terms are equal over $\mathbf{S l} * \mathbf{K}$, then either they both involve the operation $x \mapsto x^{\omega}$ or neither does. This reduces the $\kappa$-word problem for $\mathbf{S l} * \mathbf{K}$ to $\kappa$-terms involving the operation $x \mapsto x^{\omega}$. Observe at last that the following reduction rules are valid for $\mathbf{S l} * \mathbf{K}$ (i.e. the two sides of any rule coincide over $\mathbf{S l} * \mathbf{K})$ :

$$
\begin{array}{rlll}
\mathrm{r} 1) & x^{\omega} y z^{\omega} & \rightarrow x^{\omega} y z \quad x, z \neq 1, \\
\mathrm{r} 2) & x^{\omega} y z & \rightarrow x^{\omega} y & x, z \neq 1, c(z) \subseteq c(x y), \\
\mathrm{r} 3) & \left(y x^{\omega} z\right)^{\omega} & \rightarrow y x^{\omega} z y & x \neq 1, \\
\mathrm{r} 4) & \left(x^{n}\right)^{\omega} & \rightarrow x^{\omega} & x \neq 1, \\
\mathrm{r} 5) & x(y x)^{\omega} & \rightarrow(x y)^{\omega} & x \neq 1 .
\end{array}
$$

This system is Notherian since the rules reduce the length of terms. We say that a term is irreducible if it is not possible to apply a reduction rule to it. By rules $\mathrm{r} 1$ ) and r3), an irreducible term is of the form $u v^{\omega} w$ where $u, v$ and $w$ are finite words. Moreover, by rule $\mathrm{r} 2), w$ is a linear word such that $c(w) \cap c(v)=\emptyset$ and, by rule $\mathrm{r} 4), v$ is a primitive word. Finally, by rule r5), no non-trivial suffix of $u$ is a suffix of $v$. To conclude, it 
suffices to show that the equality of two irreducible terms $t_{1}=u_{1} v_{1}^{\omega} w_{1}$ and $t_{2}=u_{2} v_{2}^{\omega} w_{2}$ is decidable for $\mathbf{S l} * \mathbf{K}$. Let $u_{1}^{\prime} \in A^{*}$ be the longest prefix of $u_{1}$ such that the last letter (if it exists) of $u_{1}^{\prime}$ does not belong to $c\left(v_{1}\right)$, and let $u_{2}^{\prime}$ be the analogous word for the second term. Therefore,

$$
t_{1}=u_{1}^{\prime} u_{1}^{\prime \prime} v_{1}^{\omega} w_{1} \quad \text { and } \quad t_{2}=u_{2}^{\prime} u_{2}^{\prime \prime} v_{2}^{\omega} w_{2}
$$

for some words $u_{1}^{\prime \prime}$ and $u_{2}^{\prime \prime}$ such that $c\left(u_{1}^{\prime \prime}\right) \subseteq c\left(v_{1}\right)$ and $c\left(u_{2}^{\prime \prime}\right) \subseteq c\left(v_{2}\right)$. Notice that $u_{1}^{\prime \prime} v_{1}^{\omega}$ and $u_{2}^{\prime \prime} v_{2}^{\omega}$ are idempotents over $\mathbf{S l} * \mathbf{K}$. It follows from Theorem 2.6 that $t_{1}$ and $t_{2}$ coincide over $\mathbf{S} \mathbf{l} * \mathbf{K}$ if and only if $u_{1}^{\prime}=u_{2}^{\prime}, u_{1}^{\prime \prime}=u_{2}^{\prime \prime}, v_{1}=v_{2}$ and $c\left(w_{1}\right)=c\left(w_{2}\right)$. Therefore, the $\kappa$-word problem for $\mathbf{S l} * \mathbf{K}$ is decidable.

\section{2. $\kappa$-reducibility of $\mathrm{Sl} * \mathbf{K}$}

The proof of the $\kappa$-reducibility of $\mathbf{S l} * \mathbf{K}$ is a simple adaptation of Theorem 3.1.

Let $\gamma$ be a $(\mathbf{S l} * \mathbf{K})$-inevitable labelling of a finite graph $\Gamma$ by a finite $A$-generated semigroup $S$ and let $\psi$ be the unique morphism from $\bar{\Omega}_{A} \mathbf{S}$ to $S$ which respects the choice of generators. Hence, there is a labelling $\delta$ of $\Gamma$ by $\bar{\Omega}_{A} \mathbf{S}$ such that $\psi \circ \delta=\gamma$ and $q_{\mathbf{S l} * \mathbf{K}} \circ \delta$ is consistent. Notice that since $\mathbf{K}$ is a subpseudovariety of $\mathbf{S l} * \mathbf{K}$, this implies that $q_{\mathbf{K}} \circ \delta$ is also consistent. To prove the $\kappa$-reducibility of $\mathbf{S l} * \mathbf{K}$, we have to construct a labelling $\delta^{\prime}: \Gamma \rightarrow \Omega_{A}^{\kappa} \mathbf{S}$ such that $\psi \circ \delta^{\prime}=\gamma$ and $p_{\mathbf{S} 1 * \mathbf{K}} \circ \delta^{\prime}$ is consistent.

Using the same notations and following the construction of $\delta^{\prime}$ in Theorem 3.1, one can show that equalities (3), (4) and (5) can also be deduced for this case. Therefore, by Lemma 2.7, the factorizations (3) and (4) imply the existence of implicit operations $\pi_{\theta(\mathrm{v})}, \rho_{\mathrm{v}}$ and $\rho_{\mathrm{e}}$, with $\pi_{\theta(\mathrm{v})}$ non-explicit, such that

$$
\begin{aligned}
& \delta(\mathrm{v})=p_{\theta(\mathrm{v})} \cdot u v \cdot \pi_{\theta(\mathrm{v})} \cdot \rho_{\mathrm{v}}, \\
& \delta(\mathrm{e})=p_{\mathrm{e}} \cdot u v \cdot \pi_{\theta(\mathrm{v})} \cdot \rho_{\mathrm{e}} \text { for every edge } \mathrm{e} \in \mathcal{E}_{\theta(\mathrm{v})},
\end{aligned}
$$

where $u v \in A^{+}$is the prefix of $w_{\theta(\mathrm{v})}$ such that $|v|=|S|$ and $u \in A^{*}$ is the longest prefix of $w_{\theta(\mathrm{v})}$ such that the last letter (if it exists) of $u$ does not belong to $c_{\infty}\left(w_{\theta(\mathrm{v})}\right)$. By Lemma 2.1, if $k=|S|$ and $v=a_{1} \cdots a_{k}$, then there exists a $\kappa$-term

$$
\bar{v}=a_{1} \cdots a_{i-1}\left(a_{i} \cdots a_{j}\right)^{\omega} a_{j+1} \cdots a_{k}
$$

such that $\psi(v)=\psi(\bar{v})$. Moreover, since $A^{+}$is dense in $\bar{\Omega}_{A} \mathbf{S}$ and the content morphism is continuous, there exist words $x_{\theta(\mathrm{v})}, y_{\mathrm{v}}$ and $y_{\mathrm{e}}$ such that $c\left(\pi_{\theta(\mathrm{v})}\right)=c\left(x_{\theta(\mathrm{v})}\right), c\left(\rho_{\mathrm{g}}\right)=c\left(y_{\mathrm{g}}\right)$ and $S$ satisfies $\pi_{\theta(\mathrm{v})}=x_{\theta(\mathrm{v})}$ and $\rho_{\mathrm{g}}=y_{\mathrm{g}}$, for $\mathrm{g}=\mathrm{v}$, e. We define $\delta^{\prime}$ to be the labelling of $\Gamma$ by $\Omega_{A}^{\kappa} \mathbf{S}$ as follows:

- $\delta^{\prime}$ coincides with $\delta$ on vertices and edges labelled by explicit operations;

- $\delta^{\prime}(\mathrm{v})=p_{\theta(\mathrm{v})} u \bar{v} x_{\theta(\mathrm{v})} y_{\mathrm{v}}$ for any vertex $\vee \in \mathcal{V}_{\omega}$;

- $\delta^{\prime}(\mathrm{e})=p_{\mathrm{e}} u \bar{v} x_{\theta(\mathrm{v})} y_{\mathrm{e}}$ for any edge e $\in \mathcal{E}_{\theta(\mathrm{v})}$;

- $\delta^{\prime}(\mathrm{e})$ is any explicit operation with content $c(\delta(\mathrm{e}))$ which coincides with $\delta(\mathrm{e})$ over $S$, for any edge e $\in \mathcal{E}_{\omega}$.

By construction, $\psi \circ \delta^{\prime}=\gamma$. Now, notice that $c\left(x_{\theta(\mathrm{v})}\right)=c\left(\pi_{\theta(\mathrm{v})}\right)=c_{\infty}\left(w_{\theta(\mathrm{v})}\right)$. Therefore, by (5) and by consistency of $q_{\mathbf{S} 1 * \mathbf{K}} \circ \delta$, the consistency of $p_{\mathbf{S} 1 * \mathbf{K}} \circ \delta^{\prime}$ is an easy consequence of the second part of Lemma 2.7.

This proves the following theorem.

Theorem 4.1. The pseudovariety $\mathbf{S l} * \mathbf{K}$ is $\kappa$-tame.

\section{REDUCIBILITY OF JOINS WITH D}

In this section, we show that the property of being $\sigma$-reducible is preserved for certain joins with $\mathbf{D}$.

Let $\mathbf{V}$ be a pseudovariety. We say that $\mathbf{V}$ satisfies property $\left(\mathcal{C}_{r}\right)$ (of right-cancellation) if, for every $\pi_{1}, \pi_{2}, \rho \in \bar{\Omega}_{A} \mathbf{S}$ with $\pi_{1}$ and $\pi_{2}$ non-explicit, if $q_{\mathbf{D} \vee \mathbf{V}}\left(\pi_{1} \rho\right)=q_{\mathbf{D} \vee \mathbf{V}}\left(\pi_{2} \rho\right)$, 
then $q_{\mathbf{D} \vee \mathbf{V}}\left(\pi_{1}\right)=q_{\mathbf{D} \vee \mathbf{V}}\left(\pi_{2}\right)$. Notice that any pseudovariety $\mathbf{V}$ verifying $q_{\mathbf{V}}(\pi \rho)=q_{\mathbf{V}}(\pi)$ for every $\pi, \rho \in \bar{\Omega}_{A} \mathbf{S}$ with $\pi$ non-explicit clearly satisfies $\left(\mathcal{C}_{r}\right)$. This is the case for instance of any subpseudovariety of $\mathbf{K}$. Other examples of pseudovarieties satisfying $\left(\mathcal{C}_{r}\right)$ are: $\mathbf{C o m}$ of commutative semigroups (by the considerations of [2, pp. 91-92]); $\mathbf{J}$ of $\mathcal{J}$-trivial semigroups (by the description of the pseudoidentities satisfied by $\mathbf{J}[2]$ ); any pseudovariety $\mathbf{H}$ of groups (since $\bar{\Omega}_{A} \mathbf{H}$ satisfies the cancellation law).

Theorem 5.1. Let $\sigma$ be a non-trivial implicit signature. If $\mathbf{V}$ is a $\sigma$-reducible pseudovariety satisfying $\left(\mathcal{C}_{r}\right)$, then $\mathbf{D} \vee \mathbf{V}$ is $\sigma$-reducible.

Proof. Let $\gamma$ be a $(\mathbf{D} \vee \mathbf{V})$-inevitable labelling of a finite graph $\Gamma$ by a finite $A$-generated semigroup $S$ and let $\delta$ be a labelling of $\Gamma$ by $\bar{\Omega}_{A} \mathbf{S}$ such that $\psi \circ \delta=\gamma$ and $q_{\mathbf{D} \vee \mathbf{V}} \circ \delta$ is consistent. Therefore $q_{\mathbf{D}} \circ \delta$ and $q_{\mathbf{V}} \circ \delta$ are both consistent. We have to construct a labelling $\delta^{\prime}: \Gamma \rightarrow \Omega_{A}^{\sigma} \mathbf{S}$ such that $\psi \circ \delta^{\prime}=\gamma$ and $p_{\mathbf{D} \vee \mathbf{V}} \circ \delta^{\prime}$ is consistent.

Let $\mathcal{V}_{\omega}$ be the set of vertices $v \in \mathcal{V}(\Gamma)$ such that $\delta(\mathrm{v})$ is a non-explicit operation. Let $\phi$ be the equivalence relation on $\mathcal{V}_{\omega}$ generated by the relation

$\left\{(\mathrm{v}, \mathrm{w}) \mid \mathrm{v}, \mathrm{w} \in \mathcal{V}_{\omega}\right.$ and there is an edge e from $\mathrm{v}$ to $\mathrm{w}$ such that $\delta(\mathrm{e})$ is explicit $\}$.

For each $\vee \in \mathcal{V}_{\omega}$, let $\phi(\mathrm{v})$ be the $\phi$-class of $\mathrm{v}$ and let

- $\mathcal{E}_{\phi(\mathrm{v}), 0}$ be the set of edges e $\in \mathcal{E}(\Gamma)$ such that $\omega(\mathrm{e}) \in \phi(\mathrm{v})$ and $\delta(\mathrm{e})$ is explicit (and so also $\alpha(\mathrm{e}) \in \phi(\mathrm{v})$ );

- $\mathcal{E}_{\mathrm{v}, \omega}$ be the set of edges e $\in \mathcal{E}(\Gamma)$ such that $\omega(\mathrm{e})=\mathrm{v}$ and $\delta(\mathrm{e})$ is non-explicit.

Let $\Phi$ be a $\phi$-class such that $\mathcal{E}_{\Phi, 0}$ is non-empty and select a vertex $v_{\Phi} \in \Phi$. Let $m_{\Phi}$ be the maximal length of labels of undirected paths, having no repeated edges, consisting of edges of $\mathcal{E}_{\Phi, 0}$. Since $\delta\left(v_{\Phi}\right)$ is non-explicit, there is a factorization

$$
q_{\mathbf{D}}\left(\delta\left(\mathrm{v}_{\Phi}\right)\right)=z_{\Phi} s_{\mathrm{v}_{\Phi}}
$$

where $s_{\mathrm{v}_{\Phi}}$ is a word of length $m_{\Phi}$ and $z_{\Phi} \in \bar{\Omega}_{A} \mathbf{D}$ is a non-explicit operation. Let $\mathbf{v} \in \Phi$. Select an undirected path from $v_{\Phi}$ to $v$ consisting of edges of $\mathcal{E}_{\Phi, 0}$ with minimal length of labels. Let $h$ be the label of this path and put $s_{\mathrm{v}}=s_{\mathrm{V}_{\Phi}} h$. Since the length of $h$ is at most $m_{\Phi}$, and since the action $h$ on $s_{\mathrm{V}_{\Phi}}$ is defined, $s_{\mathrm{V}}$ belongs to $A^{*}$. By consistency of $q_{\mathbf{D}} \circ \delta$, we have

$$
q_{\mathbf{D}}(\delta(\mathrm{v}))=z_{\Phi} s_{\mathrm{v}} .
$$

Moreover, for every edge e $\in \mathcal{E}_{\mathrm{v}, \omega}$,

$$
q_{\mathbf{D}}(\delta(\mathrm{e}))=q_{\mathbf{D}}(\delta(\mathrm{v}))=z_{\Phi} s_{\mathrm{v}} .
$$

Now, we deduce from Lemma 2.5 the existence of non-explicit operations $\pi_{\Phi}, \rho_{\mathrm{v}}$ and $\rho_{\mathrm{e}}$ such that

$$
\begin{aligned}
& \delta(\mathrm{v})=\rho_{\mathrm{v}} \cdot \pi_{\Phi} \cdot s_{\mathrm{v}}, \\
& \delta(\mathrm{e})=\rho_{\mathrm{e}} \cdot \pi_{\Phi} \cdot s_{\mathrm{v}} \text { for any edge } \mathrm{e} \in \mathcal{E}_{\mathrm{v}, \omega} .
\end{aligned}
$$

Notice that for a vertex $v \in \mathcal{V}_{\omega}$, if $\mathcal{E}_{\phi(\mathrm{v}), 0}$ is the empty set, then $\phi(\mathrm{v})=\{\mathrm{v}\}$ and factorizations (12) and (13) can be deduced with $s_{\mathrm{v}}$ the empty word.

Let us notice the following observation.

Lemma 5.2. For each vertex $\vee \in \Phi$ and each edge $\mathrm{e} \in \mathcal{E}_{\mathrm{v}, \omega}, q_{\mathbf{D} \vee \mathbf{V}}\left(\rho_{\mathrm{v}_{\Phi}}\right)=q_{\mathbf{D} \vee \mathbf{V}}\left(\rho_{\mathrm{v}}\right)=$ $q_{\mathbf{D} \vee \mathbf{V}}\left(\delta(\alpha(\mathrm{e})) \cdot \rho_{\mathrm{e}}\right)$.

Proof. Let $\mathcal{P}_{\mathrm{v}}$ be the undirected path $\mathrm{e}_{1}, \mathrm{e}_{2}, \ldots, \mathrm{e}_{n}$ from $\mathrm{v}_{\Phi}$ to $v$ used in the definition of $s_{\mathrm{v}}$ and let $h$ be the label of this path, so that $s_{\mathrm{v}}=s_{\mathrm{v}_{\Phi}} h$. We prove the first equality by induction on $n$. If $n=1$, then $\mathrm{e}_{1}$ is either an edge from $\mathrm{v}_{\Phi}$ to $\mathrm{v}$ or from $\mathrm{v}$ to $\mathrm{v}_{\Phi}$. In the first case $s_{\vee}=s_{\mathrm{V}_{\Phi}} \delta\left(\mathrm{e}_{1}\right)$ and, by (12) and the fact that $q_{\mathbf{D} \vee \mathbf{V}} \circ \delta$ is consistent,

$$
q_{\mathbf{D} \vee \mathbf{V}}\left(\rho_{\mathrm{v}_{\Phi}} \pi_{\Phi} s_{\mathrm{v}_{\Phi}} \delta\left(\mathrm{e}_{1}\right)\right)=q_{\mathbf{D} \vee \mathbf{V}}\left(\rho_{\mathrm{v}} \pi_{\Phi} s_{\mathrm{v}}\right) .
$$

Therefore, $q_{\mathbf{D} \vee \mathbf{V}}\left(\rho_{\mathrm{v}_{\Phi}} \pi_{\Phi} s_{\mathrm{v}_{\Phi}} \delta\left(\mathrm{e}_{1}\right)\right)=q_{\mathbf{D} \vee \mathbf{V}}\left(\rho_{\mathrm{v}} \pi_{\Phi} s_{\mathrm{v}_{\Phi}} \delta\left(\mathrm{e}_{1}\right)\right)$. Hence, since $\mathbf{V}$ satisfies property $\left(\mathcal{C}_{r}\right)$, we deduce that $q_{\mathbf{D} \vee \mathbf{V}}\left(\rho_{\vee_{\Phi}}\right)=q_{\mathbf{D} \vee \mathbf{V}}\left(\rho_{\vee}\right)$. The second case is symmetric. 
Let now $n>1$ and assume by induction hypothesis that the lemma is true for all vertices $\mathrm{v}^{\prime}$ for which $\mathcal{P}_{\mathrm{v}^{\prime}}$ has $n-1$ edges. Let $\mathrm{v}^{\prime}$ be the end vertex of the undirected path $\mathrm{e}_{1}, \mathrm{e}_{2}, \ldots, \mathrm{e}_{n-1}$. We may assume that this path is $\mathcal{P}_{\mathrm{v}^{\prime}}$ so that $s_{\mathrm{v}}=s_{\mathrm{v}^{\prime}} \delta\left(\mathrm{e}_{n}\right)^{\epsilon}$, where $\epsilon=1$ if the edge $\alpha\left(\mathrm{e}_{n}\right)=\mathrm{v}^{\prime}$ and $\epsilon=-1$ otherwise. By induction hypothesis $q_{\mathbf{D} \vee \mathbf{V}}\left(\rho_{\mathbf{v}_{\Phi}}\right)=q_{\mathbf{D} \vee \mathbf{V}}\left(\rho_{\mathrm{v}^{\prime}}\right)$ and, as in the case $n=1$, one can prove that $q_{\mathbf{D} \vee \mathbf{V}}\left(\rho_{\mathbf{v}}\right)=$ $q_{\mathbf{D} \vee \mathbf{V}}\left(\rho_{\vee^{\prime}}\right)$. Therefore $q_{\mathbf{D} \vee \mathbf{V}}\left(\rho_{\vee_{\Phi}}\right)=q_{\mathbf{D} \vee \mathbf{V}}\left(\rho_{\vee}\right)$. The first equality follows by induction. The second equality follows from (12) and (13) and the facts that $q_{\mathbf{D} \vee \mathbf{V}} \circ \delta$ is consistent and $\mathbf{V}$ satisfies property $\left(\mathcal{C}_{r}\right)$.

Suppose now that the restriction of $\delta$ to $\mathcal{E}_{\Phi, 0}$ is not a commuting labelling. Then, by Lemma 2.5 of [9], for any vertex $\vee \in \Phi, q_{\mathbf{D}}(\delta(\mathrm{v}))=u_{\Phi}^{-\infty} v_{\mathrm{v}}$ for some words $u_{\Phi} \neq 1$ and $v_{\mathrm{v}}$, with $\left|v_{\mathrm{v}}\right| \leq m_{\Phi}$. In particular,

$$
q_{\mathbf{D}}\left(\delta\left(\mathrm{v}_{\Phi}\right)\right)=z_{\Phi} s_{\mathrm{v}_{\Phi}}=y_{\Phi}^{-\infty} s_{\mathrm{v}_{\Phi}}
$$

for a conjugate $y_{\Phi}$ of $u_{\Phi}$, and, by Lemma 2.5 , one can choose $\pi_{\Phi}=y_{\Phi}^{\omega}$. Let e $\in \mathcal{E}_{\Phi, 0}$ be an edge such that

$$
s_{\alpha(\mathrm{e})} \delta(\mathrm{e}) \neq s_{\omega(\mathrm{e})} .
$$

Since $q_{\mathbf{D}}(\delta(\alpha(\mathrm{e})) \delta(\mathrm{e}))=q_{\mathbf{D}}\left(\delta(\omega(\mathrm{e}))\right.$, we deduce that $q_{\mathbf{D}}\left(y_{\Phi}^{\omega} s_{\alpha(\mathrm{e})} \delta(\mathrm{e})\right)=q_{\mathbf{D}}\left(y_{\Phi}^{\omega} s_{\omega(\mathrm{e})}\right)$. That is,

$$
y_{\Phi}^{-\infty} s_{\alpha(\mathrm{e})} \delta(\mathrm{e})=y_{\Phi}^{-\infty} s_{\omega(\mathrm{e})} .
$$

By definition of the words $s_{\alpha(\mathrm{e})}$ and $s_{\omega(\mathrm{e})}$ (by paths with minimal lengths of labels) one deduces that $|\delta(\mathrm{e})|<\left|s_{\omega(\mathrm{e})}\right|<\left|s_{\alpha(\mathrm{e})} \delta(\mathrm{e})\right|$. Therefore, (14) implies that

$$
s_{\omega(\mathrm{e})}=x \delta(\mathrm{e}) \quad \text { and } \quad s_{\alpha(\mathrm{e})}=z x
$$

for some words $x, z \in A^{+}$. Moreover, by cancelling $s_{\omega(\mathrm{e})}$, we have $y_{\Phi}^{-\infty} z=y_{\Phi}^{-\infty}$ which implies that $z=y_{\Phi}^{k}$ for some integer $k$. Now, by consistency of $q_{\mathbf{D} \vee \mathbf{V}} \circ \delta$,

$$
q_{\mathbf{D} \vee \mathbf{V}}\left(\rho_{\alpha(\mathrm{e})} y_{\Phi}^{\omega} s_{\alpha(\mathrm{e})} \delta(\mathrm{e})\right)=q_{\mathbf{D} \vee \mathbf{V}}\left(\rho_{\omega(\mathrm{e})} y_{\Phi}^{\omega} s_{\omega(\mathrm{e})}\right) .
$$

Hence, since $\mathbf{V}$ satisfies property $\left(\mathcal{C}_{r}\right), q_{\mathbf{D} \vee \mathbf{V}}\left(\rho_{\alpha(\mathrm{e})} y_{\Phi}^{\omega} y_{\Phi}^{k}\right)=q_{\mathbf{D} \vee \mathbf{V}}\left(\rho_{\omega(\mathrm{e})} y_{\Phi}^{\omega}\right)$, so that, by Lemma 5.2, $q_{\mathbf{D} \vee \mathbf{V}}\left(\rho_{\mathrm{v}_{\Phi}} y_{\Phi}^{\omega} y_{\Phi}^{k}\right)=q_{\mathbf{D} \vee \mathbf{V}}\left(\rho_{\mathrm{v}_{\Phi}} y_{\Phi}^{\omega}\right)$, where $\mathbf{v}_{\Phi}$ is the representative of the $\phi$ class $\Phi$. Let $l \in \mathbb{N}$ be the minimal integer such that $q_{\mathbf{D} \vee \mathbf{V}}\left(\rho_{\mathbf{v}_{\Phi}} y_{\Phi}^{\omega} y_{\Phi}^{l}\right)=q_{\mathbf{D} \vee \mathbf{V}}\left(\rho_{\mathbf{v}_{\Phi}} y_{\Phi}^{\omega}\right)$. We may assume $l=1$, since otherwise we could choose $\pi_{\Phi}=\left(y_{\Phi}^{l}\right)^{\omega}$. Therefore

$$
q_{\mathbf{D} \vee \mathbf{V}}\left(\rho_{\mathrm{v}_{\Phi}} y_{\Phi}^{\omega} y_{\Phi}\right)=q_{\mathbf{D} \vee \mathbf{V}}\left(\rho_{\mathrm{v}_{\Phi}} y_{\Phi}^{\omega}\right)
$$

We extend graph $\Gamma$ to a graph $\Gamma_{1}$ and let $\gamma_{1}$ and $\delta_{1}$ be respectively the extensions of $\gamma$ and $\delta$ to $\Gamma_{1}$ as follows. For each $\phi$-class $\Phi$ write $\pi_{\Phi}=\pi_{\Phi, 1} \pi_{\Phi, 2}$ with $\pi_{\Phi, 2}$ non-explicit (if the restriction of $\delta$ to $\mathcal{E}_{\Phi, 0}$ is not a commuting labelling, let $\pi_{\Phi, 1}=\pi_{\Phi, 2}=y_{\Phi}^{\omega}$ ). Then,

- there are two vertices $\mathrm{w}_{\Phi, 1}$ and $\mathrm{w}_{\Phi, 2}$, and an edge $\mathrm{f}_{\Phi, 1}$ (from $\mathrm{w}_{\Phi, 1}$ to $\mathrm{w}_{\Phi, 2}$ ) labelled under $\delta_{1}$ by $\rho_{\mathrm{v}_{\Phi}} \pi_{\Phi, 1}, \rho_{\mathrm{v}_{\Phi}} \pi_{\Phi}$ and $\pi_{\Phi, 2}$, respectively, where $\mathrm{v}_{\Phi}$ is the vertex fixed in $\Phi$;

- for each vertex $v \in \Phi$, there is a vertex $w_{v}$ and two edges $f_{v, 1}$ (from $w_{v}$ to $w_{\Phi, 1}$ ) and $\mathrm{f}_{\mathrm{v}, 2}$ (from $\mathrm{w}_{\Phi, 2}$ to $\mathrm{v}$ ) labelled under $\delta_{1}$ by $\rho_{\mathrm{v}}, \pi_{\Phi, 1}$ and $s_{\mathrm{v}}$, respectively.

- for each edge $\mathrm{e} \in \mathcal{E}_{\mathrm{v}, \omega}$ with $\mathrm{v} \in \Phi$, there is an edge $\mathrm{f}_{\mathrm{e}}$ (from $\alpha(\mathrm{e})$ to $\mathrm{w}_{\Phi, 1}$ ) labelled under $\delta_{1}$ by $\rho_{\mathrm{e}} \pi_{\Phi, 1}$.

- if the restriction of $\delta$ to $\mathcal{E}_{\Phi, 0}$ is not a commuting labelling, there is an edge $\mathrm{f}_{\Phi, 2}$ (from $\mathrm{w}_{\Phi, 2}$ to $\mathrm{w}_{\Phi, 2}$ ) labelled under $\delta_{1}$ by $y_{\Phi}$;

- for each element $\mathrm{g} \in \Gamma_{1} \backslash \Gamma, \gamma_{1}(\mathrm{~g})=\psi\left(\delta_{1}(\mathrm{~g})\right)$.

By construction $\psi \circ \delta_{1}=\gamma_{1}$. On the other hand, by Lemma 5.2,

$$
q_{\mathbf{D} \vee \mathbf{V}}\left(\rho_{\mathrm{v}}\right)=q_{\mathbf{D} \vee \mathbf{V}}\left(\rho_{\mathrm{v}^{\prime}}\right)=q_{\mathbf{D} \vee \mathbf{V}}\left(\delta(\alpha(\mathrm{e})) \cdot \rho_{\mathrm{e}}\right)
$$

for each vertex $\vee \in \mathcal{V}_{\omega}$, each $\mathrm{v}^{\prime} \in \phi(\mathrm{v})$ and each $\mathrm{e} \in \mathcal{E}_{\mathrm{v}, \omega}$. Therefore, as $q_{\mathbf{D} \vee \mathbf{V}} \circ \delta$ is consistent, we deduce from (12), (13) and (16) that $q_{\mathbf{D} \vee \mathbf{V}} \circ \delta_{1}$ is consistent. In particular, $q_{\mathbf{V}} \circ \delta_{1}$ is consistent. Therefore, since $\mathbf{V}$ is $\sigma$-reducible there exists a labelling $\delta_{1}^{\prime}$ of $\Gamma_{1}$ 
by $\Omega_{A}^{\sigma} \mathbf{S}$ such that $\psi \circ \delta_{1}^{\prime}=\gamma_{1}$ and $p_{\mathbf{V}} \circ \delta_{1}^{\prime}$ is consistent. Moreover, by Lemma 2.3, we may assume that $\delta_{1}^{\prime}\left(\mathfrak{f}_{\Phi, 1}\right)$ is non-explicit for every $\phi$-class $\Phi$ and that $\delta_{1}^{\prime}(\mathrm{g})=\delta_{1}(\mathrm{~g})$ for every $\mathrm{g} \in \Gamma_{1}$ such that $\delta_{1}(\mathrm{~g})$ is explicit. In particular, $\delta_{1}^{\prime}(\mathrm{g})=\delta(\mathrm{g})$ for every $\mathrm{g} \in \Gamma$ such that $\delta(\mathrm{g})$ is explicit.

We define $\delta^{\prime}$ to be the labelling of $\Gamma$ by $\Omega_{A}^{\sigma} \mathbf{S}$ as follows.

1) $\delta^{\prime}$ coincides with $\delta$ on vertices and edges labelled by explicit operations;

2) Let $v \in \mathcal{V}_{\omega}$ be a vertex such that the restriction of $\delta$ to $\mathcal{E}_{\phi(v), 0}$ is a commuting labelling. Let $\Phi=\phi(\mathrm{v})$. In this case, the label of an undirected path consisting of edges of $\mathcal{E}_{\Phi, 0}$ only depends on its initial and terminal vertices. Therefore, $s_{\alpha(\mathrm{e})} \delta(\mathrm{e})=s_{\omega(\mathrm{e})}$ for each edge e $\in \mathcal{E}_{\Phi, 0}$. In this case, we define

$$
\delta^{\prime}(\mathrm{v})=\delta_{1}^{\prime}\left(\mathrm{w}_{\mathrm{v}}\right) \delta_{1}^{\prime}\left(\mathrm{f}_{\mathrm{v}, 1}\right) \delta_{1}^{\prime}\left(\mathrm{f}_{\Phi, 1}\right) \delta_{1}^{\prime}\left(\mathrm{f}_{\mathrm{v}, 2}\right)=\delta_{1}^{\prime}\left(\mathrm{w}_{\mathrm{v}}\right) \delta_{1}^{\prime}\left(\mathrm{f}_{\mathrm{v}, 1}\right) \delta_{1}^{\prime}\left(\mathrm{f}_{\Phi, 1}\right) s_{\mathrm{v}} .
$$

Moreover, for each edge e $\in \mathcal{E}_{\mathrm{v}, \omega}$, let $\delta^{\prime}(\mathrm{e})=\delta_{1}^{\prime}\left(\mathrm{f}_{\mathrm{e}}\right) \delta_{1}^{\prime}\left(\mathrm{f}_{\Phi, 1}\right) s_{\mathrm{v}}$.

3) Let now $v \in \mathcal{V}_{\omega}$ be a vertex such that the restriction of $\delta$ to $\mathcal{E}_{\phi(v), 0}$ is not a commuting labelling. Let $\Phi=\phi(\mathrm{v})$. In this case, there is an edge $\mathrm{f}_{\Phi, 2}$ in $\Gamma_{1}$ (from $\mathrm{w}_{\Phi, 2}$ to $\mathrm{w}_{\Phi, 2}$ ) labelled under $\delta_{1}$ by the explicit operation $y_{\Phi}$. Therefore, $\delta_{1}^{\prime}\left(\mathfrak{f}_{\Phi, 2}\right)=y_{\Phi}$ and, since $\mathfrak{f}_{\Phi, 2}$ is a loop in vertex $\mathbf{w}_{\Phi, 2}$ and $p_{\mathbf{V}} \circ \delta_{1}^{\prime}$ is consistent, $\mathbf{V}$ satisfies

$$
\delta_{1}^{\prime}\left(\mathrm{w}_{\Phi, 2}\right)=\delta_{1}^{\prime}\left(\mathrm{w}_{\Phi, 2}\right) y_{\Phi}=\delta_{1}^{\prime}\left(\mathrm{w}_{\Phi, 2}\right) y_{\Phi}^{n}
$$

for every positive integer $n$. Let $l$ be an integer such that $S$ satisfies $y_{\Phi}^{l}=$ $y_{\Phi}^{\omega}$ and let $z=y_{\Phi}^{l}$. Let now $\varrho\left(a_{1}, \ldots, a_{r}\right)$ be a non-explicit element of the implicit signature $\sigma$ and let $\left(w_{i}\left(a_{1}, \ldots, a_{r}\right)\right)_{i}$ be a sequence of explicit operations converging to $\varrho\left(a_{1}, \ldots, a_{r}\right)$. Then $\left(w_{i}(z, \ldots, z)\right)_{i}$ is a sequence which converges to the non-explicit $\sigma$-term $\varrho(z, \ldots, z)$ which we denote by $t_{\Phi}$. Notice that

$$
\psi\left(t_{\Phi}\right)=\psi\left(y_{\Phi}^{\omega}\right) \quad \text { and } \quad p_{\mathbf{D}}\left(t_{\Phi}\right)=p_{\mathbf{D}}\left(y_{\Phi}^{\omega}\right)
$$

We define

$$
\delta^{\prime}(\mathrm{v})=\delta_{1}^{\prime}\left(\mathrm{w}_{\mathrm{v}}\right) \delta_{1}^{\prime}\left(\mathrm{f}_{\mathrm{v}, 1}\right) \delta_{1}^{\prime}\left(\mathrm{f}_{\Phi, 1}\right) t_{\Phi} \delta_{1}^{\prime}\left(\mathrm{f}_{\mathrm{v}, 2}\right)=\delta_{1}^{\prime}\left(\mathrm{w}_{\mathrm{v}}\right) \delta_{1}^{\prime}\left(\mathrm{f}_{\mathrm{v}, 1}\right) \delta_{1}^{\prime}\left(\mathrm{f}_{\Phi, 1}\right) t_{\Phi} s_{\mathrm{v}} .
$$

Moreover, for each edge e $\in \mathcal{E}_{\mathrm{v}, \omega}$, let $\delta^{\prime}(\mathrm{e})=\delta_{1}^{\prime}\left(\mathrm{f}_{\mathrm{e}}\right) \delta_{1}^{\prime}\left(\mathrm{f}_{\Phi, 1}\right) t_{\Phi} s_{\mathrm{v}}$. Notice that

$$
\begin{aligned}
& \psi\left(\delta_{1}^{\prime}\left(\mathrm{f}_{\Phi, 1}\right) t_{\Phi}\right)= \\
= & \psi\left(\delta_{1}^{\prime}\left(\mathrm{f}_{\Phi, 1}\right)\right) \psi\left(t_{\Phi}\right) \\
= & \psi\left(y_{\Phi}^{\omega}\right) \psi\left(y_{\Phi}^{\omega}\right) \\
& \quad \text { since } \psi \circ \delta_{1}^{\prime}=\psi \circ \delta_{1}, \delta_{1}\left(\mathrm{f}_{\Phi, 1}\right)=\pi_{\Phi, 1}=y_{\Phi}^{\omega} \text { and by }(18) \\
= & \psi\left(y_{\Phi}^{\omega}\right) \\
= & \psi\left(\delta_{1}^{\prime}\left(\mathrm{f}_{\Phi, 1}\right)\right) .
\end{aligned}
$$

Therefore $\psi \circ \delta^{\prime}(\mathrm{v})=\gamma(\mathrm{v}), \psi \circ \delta^{\prime}(\mathrm{e})=\gamma(\mathrm{e})$ and, by (18) and since $t_{\Phi}$ is nonexplicit,

$$
p_{\mathbf{D}}\left(\delta^{\prime}(\mathrm{v})\right)=p_{\mathbf{D}}\left(\delta^{\prime}(\mathrm{e})\right)=p_{\mathbf{D}}\left(t_{\Phi} s_{\mathrm{v}}\right)=p_{\mathbf{D}}\left(y_{\Phi}^{\omega} s_{\mathrm{v}}\right)
$$

for every edge $\mathrm{e} \in \mathcal{E}_{\mathrm{v}, \omega}$.

By construction, it is clear that $\delta^{\prime}$ is a labelling of $\Gamma$ by $\Omega_{A}^{\sigma} \mathbf{S}$ such that $\psi \circ \delta^{\prime}=\gamma$ and, since $p_{\mathbf{V}} \circ \delta_{1}^{\prime}$ is consistent, that $p_{\mathbf{V}} \circ \delta^{\prime}$ is consistent. Let us now show that $p_{\mathbf{D}} \circ \delta^{\prime}$ is also consistent. Indeed, it is clear that the equality $p_{\mathbf{D}}\left(\delta^{\prime}(\alpha(\mathrm{e}))\right) \cdot p_{\mathbf{D}}\left(\delta^{\prime}(\mathrm{e})\right)=p_{\mathbf{D}}\left(\delta^{\prime}(\omega(\mathrm{e}))\right)$ holds for edges e such that $\delta(\mathrm{e})$ is non-explicit or such that $\delta(\omega(\mathrm{e}))$ is explicit. Let now e be an edge such that $\delta(\mathrm{e})$ is explicit and $\omega(\mathrm{e}) \in \mathcal{V}_{\omega}$. Then $\alpha(\mathrm{e}) \in \mathcal{V}_{\omega}$ and $\phi(\alpha(\mathrm{e}))=\phi(\omega(\mathrm{e}))$. Let $\Phi=\phi(\alpha(\mathrm{e}))$. If $s_{\alpha(\mathrm{e})} \delta(\mathrm{e})=s_{\omega(\mathrm{e})}$, then it is also clear that the equality above holds. If $s_{\alpha(\mathrm{e})} \delta(\mathrm{e}) \neq s_{\omega(\mathrm{e})}$, then the restriction of $\delta$ to $\mathcal{E}_{\phi(\mathrm{v}), 0}$ is not a 
commuting labelling. Moreover, by (15) and the arguments following it, $s_{\omega(\mathrm{e})}=x \delta(\mathrm{e})$ and $s_{\alpha(\mathrm{e})}=y_{\Phi}^{k} x$ with $x \in A^{+}$and $k \in \mathbb{N}$. Then

$$
\begin{aligned}
& p_{\mathbf{D}}\left(\delta^{\prime}(\alpha(\mathrm{e}))\right) \cdot p_{\mathbf{D}}\left(\delta^{\prime}(\mathrm{e})\right)= \\
= & p_{\mathbf{D}}\left(y_{\Phi}^{\omega} s_{\alpha(\mathrm{e})} \delta(\mathrm{e})\right) \quad \text { by }(19) \text { and since } \delta^{\prime}(\mathrm{e})=\delta(\mathrm{e}) \\
= & p_{\mathbf{D}}\left(y_{\Phi}^{\omega} y_{\Phi}^{k} x \delta(\mathrm{e})\right) \\
= & p_{\mathbf{D}}\left(y_{\Phi}^{\omega} s_{\omega(\mathrm{e})}\right) \quad \text { since } \mathbf{D} \text { satisfies the pseudoidentity } x^{\omega} x=x^{\omega} \\
= & p_{\mathbf{D}}\left(\delta^{\prime}(\omega(\mathrm{e}))\right) \text { again by }(19) .
\end{aligned}
$$

This proves that $p_{\mathbf{D}} \circ \delta^{\prime}$ is consistent. As $p_{\mathbf{V}} \circ \delta^{\prime}$ is also consistent, we deduce that $p_{\mathbf{D} \vee \mathbf{V}} \circ \delta_{1}^{\prime}$ is consistent. This concludes the proof of the theorem.

As a consequence of this theorem, we deduce that $\sigma$-tameness is preserved under joins with $\mathbf{D}$ for pseudovarieties satisfying $\left(\mathcal{C}_{r}\right)$.

Corollary 5.3. If $\mathbf{V}$ is a pseudovariety satisfying $\left(\mathcal{C}_{r}\right)$ which is tame with respect to a non-trivial implicit signature $\sigma$ and the $\sigma$-word problem for $\mathbf{D}$ is decidable, then $\mathbf{D} \vee \mathbf{V}$ is also $\sigma$-tame. In particular, $\mathbf{D} \vee \mathbf{V}$ is $\kappa$-tame for every $\kappa$-tame pseudovariety $\mathbf{V}$ satisfying $\left(\mathcal{C}_{r}\right)$.

So, for instance, $\mathbf{D} \vee \mathbf{G}, \mathbf{D} \vee \mathbf{A b}$ and $\mathbf{D} \vee \mathbf{J}$ are $\kappa$-tame.

Since $\mathbf{L I}=\mathbf{K} \vee \mathbf{D}$, we deduce from the results of Section 3 that the results of this section hold with $\mathbf{D}$ replaced by $\mathbf{L I}$.

\section{ACKNOWLEDGEMENTS}

I would like to thank Marc Zeitoun for an observation which permitted to establish the last statement of Lemma 2.3 and helped to simplify a first version of this paper.

\section{REFERENCES}

[1] Albert, D.; Baldinger, R.; Rhodes, J. Undecidability of the identity problem for finite semigroups. J. Symbolic Logic 1992, 57 (1), 179-192.

[2] Almeida, J. Finite semigroups and universal algebra; World Scientific: Singapore, 1995.

[3] Almeida, J. Hyperdecidable pseudovarieties and the calculation of semidirect products. Int. J. Algebra and Computation 1999, 9, 241-261.

[4] Almeida, J. Finite semigroups: An Introduction to a unified theory of pseudovarieties. In Semigroups, Algorithms, Automata and Languages; Gomes, G., Pin, J.-E., Silva, P., Eds.; World Scientific: New Jersey/London/Singapore/Hong Kong, 2002; 3-64.

[5] Almeida, J.; Delgado, M. Tameness of the pseudovariety of abelian groups, Technical Report CMUP 2001-24; Univ. Porto: Porto, 2001.

[6] Almeida, J.; Steinberg, B. On the decidability of iterated semidirect products and applications to complexity. Proc. London Math. Soc. 2000, 80, 50-74.

[7] Almeida, J.; Weil, P. Free profinite semigroups over semidirect products. Russian Math. (Iz. VUZ) 1995, 39, 1-27.

[8] Almeida, J.; Zeitoun, M. The pseudovariety $\mathbf{J}$ is hyperdecidable. Theoretical Informatics and Applications 1997, 31, 457-482.

[9] Almeida, J.; Zeitoun, M. Tameness of some locally trivial pseudovarieties. Comm. Algebra, to appear.

[10] Costa, J. C. Some pseudovariety joins involving locally trivial semigroups. Semigroup Forum 2002, 64, 12-28.

[11] Costa, J. C.; Teixeira, M. L. Tameness of the pseudovariety LSl, Technical Report CMAT 6-2002; Univ. Minho: Braga, 2002.

[12] Costa, J. C.; Zeitoun, M. Tameness and joins of pseudovarieties, in preparation.

[13] Reiterman, J. The Birkhoff theorem for finite algebras. Algebra Universalis 1982, 14, 1-10.

[14] Rhodes, J. Undecidability, automata and pseudovarieties of semigroups, Int. J. Algebra and Computation 1999, 9, 455-473. 